\title{
Quantum dot defined in two-dimensional electron gas at n-AlGaAs/GaAs heterojunction: simulation of electrostatic potential and charging properties
}

\author{
S. Bednarek, K. Lis, and B. Szafran \\ Faculty of Physics and Applied Computer Science, \\ AGH University of Science and Technology, \\ al. Mickiewicza 30, 30-059 Kraków, Poland
}

(Dated: October 27, 2018)

\begin{abstract}
We present a self-consistent Schroedinger-Poisson scheme for simulation of electrostatic quantum dots defined in gated two-dimensional electron gas formed at n-AlGaAs/GaAs heterojunction. The computational method is applied to a quantitative description of transport properties studied experimentally by Elzermann et al. [Appl. Phys. Lett. 84, 4617 (2004)]. The three-dimensional model describes the electrostatics of the entire device with a quantum dot that changes shape and floats inside a gated region when the applied voltages are varied. Our approach accounts for the metal electrodes of arbitrary geometry and configuration, includes magnetic field applied perpendicular to the growth direction, electron-electron correlation in the confined electron system and its interaction with the electron reservoir surrounding the quantum dot. We calculate the electric field, the space charge distribution as well as energies and wave functions of confined electrons to describe opening of two transport channels between the reservoir and the confined charge puddle. We determine the voltages for charging the dot with up to 4 electrons. The results are in a qualitative and quantitative agreement with the experimental data.
\end{abstract}

PACS numbers: 73.21.La 


\section{INTRODUCTION}

A quest for a nanodevice that would store a quantum bit in the spin 122 of a confined electron and allow for its manipulation is a main factor that motivates the research on gated electrostatic quantum dots. Realization of a spin quantum gate requires application of multiple quantum dots coupled in way that can be controlled during the device operation. First electrostatic quantum dots were formed in a vertical configuration $3,4,5,6,7$ of a semiconductor heterostructure containing a single or multiple quantum well surrounded by a single gate creating the lateral confinement. The strength of the tunnel coupling between the vertical dot and electron reservoirs depends on the applied barrier thickness and composition, and therefore it is fixed for each device. Similarly, for vertical artificial molecules the interdot coupling is defined at the production stage.

A full control of the interdot coupling is possible in quantum dots formed in gated twodimensional electron gas. $9,10,11,12,13,14,15$ These structures are produced by deposition of multiple gates on top of the n-AlGaAs/GaAs structure containing a two-dimensional electron gas at the heterojunction. The system of gates is designed to locally deplete the electron gas in order to tailor a quantum dot of the surrounding electron reservoir. The voltages applied to the multiple gates define the confinement potential for the trapped electrons and control the tunnel barrier between the dot and the reservoir. Thus, the coupling of the confined artificial atom to the environment can be intentionally tuned from an open to a closed dot regime. For nearly open dots the Kondo effect and co-tunneling phenomena are observed. ${ }^{17}$ Coupled dots with voltage tunable interdot barriers are also produced. ${ }^{14}, 15,16$ Quantum dots formed in the gated two-dimensional electron gas are used for investigation of spin dependent transport $\frac{18}{}$ and confined spin relaxation. ${ }^{19}$ A capacitive coupling between the dot and a quantum point contact defined in the same structure $9,10,11,12,13$ is used to probe the confined states by the conductance measurements. The purpose of the present paper is to provide a theoretical description of a quantum dot formed in the gated two-dimensional electron gas. We focus our attention on the nanodevice that is probed by the quantum point contact as described in Refs. [10] and [11].

The electrostatic confinement potential in vertical quantum dots was thoroughly studied in Refs. $[20,21,22,23,24,25,26,27]$. Less attention was paid to dots based on the gated twodimensional electron gas. In particular, a theory for a double planar $\operatorname{dot}^{15}$ was provided 
in Refs. $\left.{ }^{[28}\right]$ and $\left[{ }^{29}\right]$. Ref. $[\underline{16}]$ describes a structure of a triple quantum dot. Theoretical modeling of planar dots is for several reasons more difficult than modeling of the vertical structures. In vertical dots the electrons are confined inside a relatively deep quantum well with the lateral confinement strength controlled by a single gate. In planar structures the confinement is entirely due to the voltages that are applied to multiple gates and the formed potential cavity is typically shallow. Therefore, both the confinement potential and the few-electron eigenproblem have to be calculated with a special care. Moreover, as we show below, the gates not only fix the strength of the dot confinement but also change the shape of confined charge island which floats with the voltages inside the gated area. Interaction of the confined electrons with the reservoirs is also more complex due to variation of the depleted gas region with the applied potentials. The vertical $\operatorname{dots}^{20}, 21,22,23,24,25,26$ often have circular symmetry, ${ }^{32}$ which is not the case for the planar structure discussed below.

\section{THEORY}

\section{A. Model of the structure}

The nanodevice ${ }^{10,11}$ which we aim to describe is constructed on a basis of a planar semiconductor heterostructure of $\mathrm{GaAs} / \mathrm{n}-\mathrm{Al}_{0.3} \mathrm{Ga}_{0.7} \mathrm{As}$ in which the two-dimensional electron gas is created at the GaAs side of the junction. A cross section of the layer structure is presented in Fig. 1. On the substrate side there is a $1500 \mathrm{~nm}$ thick layer of undoped GaAs with a blocking AlGaAs barrier deposited on top. Lower part of the barrier (20 nm thick) is undoped and the upper $(65 \mathrm{~nm})$ is heavily doped with donors. On top of the barrier there is a $5 \mathrm{~nm}$ thin layer of $\mathrm{n}$-doped GaAs. The donor states in AlGaAs stay $200 \mathrm{meV}$ above the conduction band minimum of GaAs. Therefore, the electrons pass to the GaAs layer but stay localized under the barrier due to the Coulomb attraction by the charge of ionized donors.

In our model we assume the shape of gates deposited on top of the structure (see Fig. 2) according to Refs. $[\underline{10}, 11]$. A negative voltage applied to the electrodes depletes the electron gas underneath and forms a lateral confinement potential in the center of the gated area. For properly adjusted voltages a few electrons stay localized in the center of the structure forming an artificial atom. The electrons are confined in the vertical direction by the barrier 


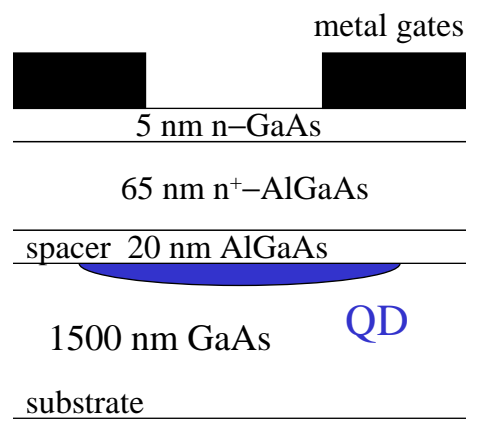

FIG. 1: (color online) Structure of layers used for formation of a gated quantum dot in the twodimensional electron gas (according to Ref. [10] ).

formed due to the GaAs/AlGaAs conduction band offset $U_{b}$. The electrostatic potential $\phi_{\text {elst }}(\mathbf{r})$ is responsible for the lateral confinement as well as for the potential that closes the dot from the substrate side. The quantum dot confinement potential is therefore expressed by

$$
U_{\text {conf }}(\mathbf{r})=U_{b}(z)-|e| \phi_{\text {elst }}(\mathbf{r})
$$

$U_{b}$ equals zero in GaAs layer and the conduction band offset in the AlGaAs barrier. The electrons are additionally confined by an in-plane magnetic field of $10 \mathrm{~T}$ applied parallel to the surface of the layer structure ${ }^{9}, 10$ Application of a strong magnetic field within the plane of confinement ${ }^{-}$was previously discussed in context of tuning (reduction) of the electron tunnel coupling for vertical artificial molecules. To the best of our knowledge the SchroedingerPoisson problem for the two dimensional electron gas with an in-plane orientation of the magnetic field was never solved previously.

\section{B. Sources of electrostatic potential}

The total electrostatic potential $\phi_{\text {tot }}(\mathbf{r})$ is influenced by voltages applied between the substrate and metal electrodes on top of the structure as well as by the charge distribution inside the device. The charge density $\rho_{\text {tot }}(\mathbf{r})$ is a sum of three contributions that have different nature and distribution

$$
\rho_{\text {tot }}(\mathbf{r})=\rho_{\text {eqd }}(\mathbf{r})+\rho_{\mathrm{d}}(\mathbf{r})+\rho_{\text {el }}(\mathbf{r}) .
$$

The first contribution $\rho_{\text {eqd }}(\mathbf{r})$ is the distribution of the charge confined in the quantum dot that is found by the solution of a few-electron quantum eigenproblem that is in the present 
work obtained by the configuration interaction method. The second contribution $\rho_{\mathrm{d}}(\mathbf{r})$ is the ionized donor space charge in the AlGaAs barrier, and the third $\rho_{\mathrm{el}}(\mathbf{r})$ is the charge density of the elntmon mon

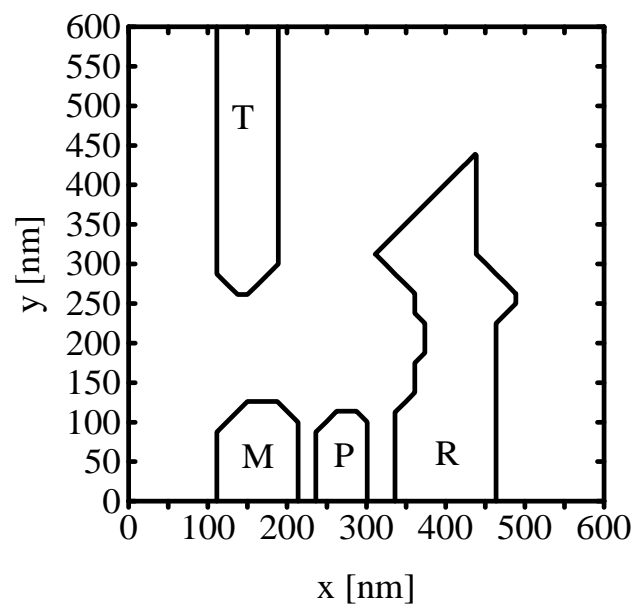

FIG. 2: Geometry, size and position of the metal gates deposited on top of the semiconductor heterostructure (according to Ref. $[\underline{10}]$ ).

According to the superposition principle the total electrostatic potential $\phi_{\text {tot }}(\mathbf{r})$ can be expressed as a sum of contributions of all the charge densities. We separate the potential due to the confined electrons $\phi_{\text {eqd }}$ of the total potential. In this way we obtain a component of the potential $\phi_{\text {elst }}$ that enters the formula (1) for the confinement potential of the electrostatic dot

$$
\phi_{\text {tot }}(\mathbf{r})=\phi_{\text {eqd }}(\mathbf{r})+\phi_{\text {elst }}(\mathbf{r})
$$

Potential of the dot-confined charge is calculated directly from the Coulomb law

$$
\phi_{\text {eqd }}(\mathbf{r})=\frac{1}{4 \pi \epsilon \epsilon_{0}} \int \frac{\rho_{\text {eqd }}\left(\mathbf{r}^{\prime}\right)}{\left|\mathbf{r}-\mathbf{r}^{\prime}\right|} d \mathbf{r}^{\prime}
$$

Potential $\phi_{\text {elst }}$ of all the other sources is found from the Poisson equation

$$
\nabla^{2} \phi_{\text {elst }}(\mathbf{r})=-\frac{\rho(\mathbf{r})}{\epsilon \epsilon_{0}}
$$

The proper boundary conditions are naturally given for the total potential $\phi_{\text {tot }}(\mathbf{r})$. The boundary conditions for the Poisson equation (5) are obtained from boundary conditions that are known for the total potential $\phi_{\text {tot }}(\mathbf{r})$ by a simple subtraction

$$
\phi_{\text {elst }}(\mathbf{r})=\phi_{\text {tot }}(\mathbf{r})-\phi_{\text {eqd }}(\mathbf{r})
$$


Charge density $\rho(\mathbf{r})$ in Eq. (5) is a sum of the charge densities of ionized donors and of the electron gas

$$
\rho(\mathbf{r})=\rho_{\mathrm{d}}(\mathbf{r})+\rho_{\mathrm{el}}(\mathbf{r})
$$

The donors are ionized in the spatial region where the total electron potential energy calculated with respect to GaAs layer is larger than the donor binding energy. For the donor energy level taken as the reference energy we obtain the ionization condition

$$
\rho_{\mathrm{d}}(\mathbf{r})=\left\{\begin{array}{ll}
0 & \text { for }-|e| \phi_{\mathrm{tot}}(\mathbf{r}) \leq E^{D} \\
|e| n_{\mathrm{D}}(\mathbf{r}) & \text { for }-|e| \phi_{\mathrm{tot}}(\mathbf{r})>E^{D}
\end{array},\right.
$$

where $n_{\mathrm{D}}(\mathbf{r})$ is the density of donor impurities, and $E^{D}$ is the donor binding energy. Assumption of a homogenous (continuous) donor distribution is justified by the presence of an undoped AlGaAs buffer (see Fig. 1).

Calculation of $\rho_{\mathrm{el}}(\mathbf{r})$ - the second charge density entering Eq. (17) that corresponds to the electron gas confined at the heterojunction - is a nontrivial task. It can be exactly evaluated only in the asymptotic region, i.e. at a large distance of the electrodes. In the neighborhood of the electrodes one has to introduce an approximate treatment (see II.D).

\section{Potential and charge distribution in the asymptotic region}

At a large distance of the electrodes the electric field is parallel to the growth direction $(z)$ and the electrostatic potential does not depend on the other two coordinates. The potential distribution results from an equilibrium between the ionized donors in the AlGaAs layer and the electron gas confined below. We choose the $y$ axis as parallel to the external magnetic field. We adapt the Landau gauge

$$
\mathbf{A}(\mathbf{r})=(-B z, 0,0)
$$

which leads to the Hamiltonian of an electron confined at the heterojunction

$$
\begin{aligned}
H(\mathbf{r})= & -\frac{\hbar^{2}}{2 m}\left(\frac{\partial^{2}}{\partial x^{2}}+\frac{\partial^{2}}{\partial y^{2}}+\frac{\partial^{2}}{\partial z^{2}}\right)+i \hbar \omega_{c} z \frac{\partial}{\partial x} \\
& +\frac{m}{2} \omega_{c}^{2} z^{2}+U_{b}(z)-|e| \phi_{\text {elst }}(z),
\end{aligned}
$$

where $\omega_{c}=\frac{|e| B}{m}$ and $m$ is the electron band mass. Since this Hamiltonian commutes with momentum in both $x$ and $y$ directions its eigenfunctions are expected to be of form

$$
\Psi^{\mathrm{as}}(\mathbf{r})=C \exp (i q x) \exp (i k y) \phi^{\mathrm{as}}(z) .
$$


Eigenequation for $\phi^{\text {as }}(z)$ is obtained by substitution of Eq. (11) into (10)

$$
H(z) \phi_{n q}^{\mathrm{as}}(z)=\varepsilon_{n q} \phi_{n q}^{\mathrm{as}}(z)
$$

where

$$
H(z)=-\frac{\hbar^{2}}{2 m} \frac{\partial^{2}}{\partial z^{2}}+\frac{m}{2} \omega_{c}^{2}\left(z-z_{0}\right)^{2}+U_{b}(z)-|e| \phi_{\mathrm{elst}}^{\mathrm{as}}(z)
$$

with $z_{0}=\hbar q / m \omega_{c}$.

Eigenvalues $\varepsilon_{n q}$ and the eigenfunctions $\phi_{n q}^{\text {as }}(z)$ are labeled by a natural quantum number $n$ and depend on the wave vector $q$ in the direction perpendicular to the magnetic field direction. Wave vector $q$ enters the Hamiltonian operator (13) through shifted harmonic oscillator minimum $z_{0}$. The total electron energy eigenvalues are given by

$$
E_{n k q}=\varepsilon_{n q}+\frac{\hbar^{2} k^{2}}{2 m}
$$

The electrons confined at the heterojunction have energies below the Fermi energy. In the present calculations the Fermi energy is taken as the reference energy level. Only the states with $E_{n k q}<0$ can be confined at the heterojunction. Given the $H(z)$ eigenfunctions one calculates the charge density of the electron gas

$$
\begin{aligned}
\rho_{\mathrm{el}}^{\mathrm{as}}(z)= & -2|e| \sum_{n k q}^{E_{n k q}<0}\left|\Psi_{n k q}^{\mathrm{as}}(x, y, z)\right|^{2} \\
& =-\sum_{n} \frac{|e|}{2 \pi^{2}} \int_{E_{n k q}<0} d k d q\left|\phi_{n q}^{\mathrm{as}}(z)\right|^{2} \\
& =-\sum_{n} \frac{|e|}{2 \pi^{2}} \int_{\varepsilon_{n q}<0} d q \int_{-k_{F}}^{k_{F}} d k\left|\phi_{n q}^{\mathrm{as}}(z)\right|^{2},
\end{aligned}
$$

where $k_{F}=\sqrt{-\frac{2 m \varepsilon_{n q}}{\hbar^{2}}}$. By integration over $k$ we obtain

$$
\rho_{\mathrm{el}}^{\mathrm{as}}(z)=-\sum_{n} \frac{|e|}{\pi^{2}} \int_{\varepsilon_{n q}<0} d q \sqrt{-\frac{2 m \varepsilon_{n q}}{\hbar^{2}}}\left|\phi_{n q}^{\mathrm{as}}(z)\right|^{2} .
$$

Apart from the electron gas another source of the electric field is the ionized donor distribution $\rho_{\mathrm{d}}$ calculated according to Eq. (8) in which we identify $\phi_{\text {tot }}^{\text {as }}=\phi_{\text {elst }}^{\text {as }}$ since at an asymptotically large distance of the quantum dot potential $\phi_{\text {eqd }}$ vanishes. Finally, the electrostatic potential is calculated from a single-dimensional Poisson equation

$$
\frac{\partial^{2}}{\partial z^{2}} \phi_{\mathrm{elst}}^{\mathrm{as}}(z)=-\frac{\rho_{\mathrm{el}}^{\mathrm{as}}(z)+\rho_{\mathrm{d}}(z)}{\epsilon \epsilon_{0}} .
$$


Since $\rho_{\mathrm{el}}^{\text {as }}$ appearing in Eq. (17) is calculated with the Schroedinger equation (12), which in turn contains the electrostatic potential, both the equations are solved in an iteration till self-consistency is reached. Eq. (12) is solved below the barrier in a computational box long enough to allow the electron charge density (16) to vanish before its end. The equilibrium solution is obtained when two conditions are met. The first one results from the charge neutrality which requires that the number of ionized donors is equal to the number of electrons trapped at the interface, which results in vanishing electric field at both ends of the region where the Poisson equation (17) is solved. In fact, whenever a self-consistency of Eq. (12) and (17) is obtained the potential derivatives at both ends of the computational box vanish. The second equilibrium condition requires that the number of ionized donors is such that the variation of the electric potential on the entire space charge (on both sides of the heterojunction) equalizes the jump in the conduction band at the GaAs/AlGaAs interface. The latter is due to the fact that the electrons occupy all the states below the Fermi energy. This includes both the donor states in the barrier (not all the donors are ionized) and the states trapped below the barrier.

The surface density of the electron charge accumulated below the barrier is obtained by integration of $\rho_{\mathrm{el}}^{\mathrm{as}}(z)$ along the growth direction

$$
\sigma^{\mathrm{as}}=\int_{-\infty}^{\infty} d z \rho_{\mathrm{el}}^{\mathrm{as}}(z)
$$

with the charge neutrality condition

$$
\sigma_{D}^{\mathrm{as}}=-\sigma^{\mathrm{as}}
$$

where $\sigma_{D}^{\text {as }}$ is the surface density of ionized donors. The surface densities depend on the conduction band offset. Under the assumption of a homogenous donor distribution the asymptotic ionization range $d$ fulfills the condition $\sigma_{D}^{\text {as }}=n_{D} d$. The second equilibrium condition is obtained for a properly chosen $d$. For a nominal composition of the barrier $\mathrm{Al}_{x} \mathrm{Ga}_{1-x} \mathrm{As}$ with $x=0.27$ the barrier height is $U_{b}=229 \mathrm{meV}$. For that value one obtains the asymptotic surface density of $\sigma^{\text {as }}=3.5 \times 10^{-11} \mathrm{~cm}^{-2}$, which is close to the nominal experimental value $\mathrm{e}^{9}$ of $2.9 \times 10^{-11} \mathrm{~cm}^{-2}$. The deviation of the calculated density off the nominal value may result from the neglect of the exchange interaction in the electron gas. We decided to reduce the barrier height to $U_{b}=200 \mathrm{meV}$ for which the calculated density is equal to its nominal value. 


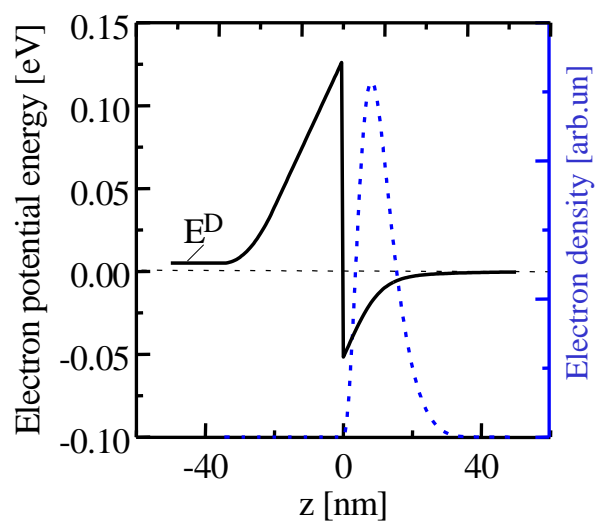

FIG. 3: Electron potential energy (solid line) and the charge density of the electron gas (dashed curve) at the AlGaAs/GaAs heterojunction (GaAs is at the positive side of $z$ ). Thin horizontal dashed line shows the Fermi energy pinned at the donor impurity level in AlGaAs. $E^{D}$ is the donor binding energy that enters formula (8).

The potential and the electron density calculated for the asymptotic region according to the procedure explained above is presented in Fig. 3. From the potential dependence on $z$ we can see that the charge neutrality condition (zero electric field at both ends of the box) is fulfilled. We also notice that the electrostatic potentials of both sides of the junction differ exactly by the donor binding energy. This is because in the discussed structure the donor impurity level in the heavily doped AlGaAs layer defines the Fermi energy.

\section{Potential and charge distribution near the quantum dot}

Under the electrodes the electron potential energy is still positive also below the AlGaAs barrier. This removes electron gas from the region below the electrodes. The electron potential energy decreases with the growing distance of the gates and it eventually becomes negative in the region where the electron gas is not completely depleted. The spatial variation of the electron density is crucial for the shape and width of the potential barriers which separate the quantum dot of the electron reservoirs. An account for the electron dependence on the potential energy is taken in a following approximate manner. We assume that the electron gas density is zero wherever the local potential energy exceeds the Fermi energy $\left(E_{F}=0\right)$. In region where the local potential energy is negative we assume that the electron 
density is proportional to its absolute value

$$
\rho_{\mathrm{el}}(x, y, z)=\left\{\begin{array}{ll}
0 & \text { for } \tau \leq 0 \\
\tau \rho_{\mathrm{el}}^{\mathrm{as}}(z) & \text { for } \tau>0
\end{array},\right.
$$

for

$$
\tau=\frac{-|e| \phi_{\mathrm{tot}}\left(x, y, z_{c}\right)+U_{b}\left(z_{c}\right)}{-|e| \phi_{\mathrm{tot}}^{\mathrm{as}}\left(z_{c}\right)+U_{b}\left(z_{c}\right)}
$$

with $\tau \leq 1$, where $\phi_{\mathrm{tot}}^{\text {as }}=\phi_{\mathrm{elst}}^{\text {as }}$ and $z_{c}$ is the center of mass of the asymptotic electron density

$$
z_{c}=\frac{1}{\sigma^{\mathrm{as}}} \int d z \rho_{\mathrm{el}}^{\mathrm{as}}(z) z
$$

The adopted formula (20) simulates the depletion of the electron gas in the region of positive potential energy. In the asymptotic region $\tau$ tends to 1, which guarantees that the known value of the electron density is found far away from the gates.

\section{E. Boundary conditions for Poisson equation in three dimensions}

Poisson equation (5) is solved in a three dimensional rectangular region which contains the quantum dot and a sufficiently large part of the electrodes. A standard test for the acceptable size of the box consists in performing the calculation in function of the box dimensions. We find that the results eventually saturate for a rectangular box of side lengths $L_{x}=L_{y}=600$ $\mathrm{nm}$ and $L_{z}=400 \mathrm{~nm}$. The position of the adopted box with respect to the electrodes is

presented in Fig. 2. In the growth direction $(z)$ the box covers $200 \mathrm{~nm}$ on both sides of the AlGaAs/GaAs heterojunction.

A unique solution of the Poisson equation is obtained for boundary condition given on the surface of the computational box and on the metal electrodes that are inside the integration region. The boundary conditions are naturally given for $\phi_{\text {tot }}$. We calculate the conditions for $\phi_{\text {elst }}$ according to Eq. (6). On the electrodes the total potential is constant and determined by applied voltages. On the surface of the electrodes we assume Dirichlet boundary conditions

$$
\phi_{\text {tot }}=U_{X}+U_{S}
$$

where $X=P, T, M$ and $R$ enumerates the electrodes (see Fig. 2), $U_{X}$ is the applied voltage and $U_{S}$ is the Schottky potential which at the metal/GaAs contact is $U_{S}=-0.65 \mathrm{~V}$. On the lateral sides of the computational box we apply Neumann boundary conditions

$$
\mathbf{n} \cdot \nabla \phi_{\text {tot }}=0
$$


where $\mathbf{n}$ is the vector normal to the surface. The Neumann conditions are consistent with the Gauss law for charge neutrality of the computational box content. On the vertical walls

of the box (parallel to the growth direction $z$ ) this boundary condition is equivalent to the assumption that the electric field is perpendicular to the heterojunction, which agrees with the boundary condition used in the asymptotic region.

\section{F. Electrons confined in the quantum dot}

The potential minimum that is found in the central region between the electrodes traps several electrons provided that the applied voltages are not too negative. Hamiltonian for the system of $N$ electrons with Landau gauge writes

$$
\begin{aligned}
H_{N}= & \sum_{i=1}^{N}\left(-\frac{\hbar^{2}}{2 m} \nabla_{i}^{2}+i \hbar \omega_{c}\left(z_{i}-z_{o}\right) \frac{\partial}{\partial x_{i}}\right. \\
& \left.+\frac{m}{2} \omega_{c}^{2}\left(z_{i}-z_{o}\right)^{2}+U_{b}(z)-|e| \phi_{\text {elst }}\left(\mathbf{r}_{i}\right)\right) \\
& +\sum_{j=1}^{N} \sum_{i>j}^{N} \frac{e^{2}}{4 \pi \epsilon \epsilon_{0}\left|\mathbf{r}_{i}-\mathbf{r}_{j}\right|} .
\end{aligned}
$$

The eigenproblem $H_{N} \Psi_{N}=E_{N} \Psi_{N}$ is solved with a configuration interaction approach in the basis of Slater determinants built of single-electron wave functions that are calculated with a finite-difference technique on a three-dimensional mesh. The convergence for the ground state energy of 4 electrons is achieved for the basis containing all the Slater determinants that can be constructed of the wave functions of 10 lowest-energy single-electron levels.

The parameter $z_{o}$ introduced by the gauge transformation is taken to minimize the total energy of $N$ electrons. In our calculations we adopt $z_{o} \simeq 12 \mathrm{~nm}$ which coincides with the center of the density of the dot-confined electrons ${ }^{33}$ (see also Fig. 2).

The few-electron wave function is used to evaluate the confined charge density

$$
\rho_{\text {eqd }}\left(\mathbf{r}_{1}\right)=-|e| \int d \mathbf{r}_{2} d \mathbf{r}_{3} \ldots d \mathbf{r}_{N}\left|\Psi_{N}\left(\mathbf{r}_{1}, \mathbf{r}_{2}, \mathbf{r}_{3}, \ldots, \mathbf{r}_{N}\right)\right|^{2}
$$

The ground-state energies for $N$ and $N-1$ electrons determine the electrochemical potential of the $N$-electron quantum dot

$$
\mu_{N}=E_{N}-E_{N-1}
$$

The dot is filled with exactly $N$ electrons when $\mu_{N}<E_{F}<\mu_{N+1}$. Charging lines that are detected in the experiment correspond to $\mu_{N}=E_{F}$. 


\section{G. Numerical procedure and self-consistency}

The Poisson equation (5) is solved on a three dimensional mesh with a finite difference method. The adopted mesh steps $\Delta x=\Delta y=12.5 \mathrm{~nm}$ and $\Delta z=2 \mathrm{~nm}$ are sufficient to describe the charge distributions and the shapes of electrodes. Smaller step in the growth direction is necessary because of a strong localization of the electron gas at the interface. Same step sizes are applied in the Schroedinger equation.

According to expression (7) the charge density that enters the Poisson equation (5) is a sum of charge densities of ionized donors that depend on the potential in a manner defined by Eq. (8) and the electron gas accumulated at the GaAs/AlGaAs interface. The latter depends on the total potential according to Eq. (20). Electrons trapped by the quantum dot are the third charge density [Eq.(26)] present in the nanodevice. The Schroedinger-Poisson calculations are iterated till the self-consistency of the three charge distributions with the total potential is obtained. The iteration accounting for the dependence of the electrons and ionized donors on the electrostatic potential requires application of an under-relaxation technique to ensure stability. The under-relaxed iteration is usually slowly convergent and requires several hundred thousands iteration for the entire mesh. The convergence is radically improved for a strategy of simulated cooling of the system. The measurements are performed at temperatures of several mK, for which occupation of electron states in Eqs. (8) and (20) has a nearly binary distribution. For non-zero temperatures we replace the formulas for the distribution of ionized donors and the electron gas used in the above theory by expressions accounting for the Fermi statistics

$$
\rho_{\mathrm{d}}(\mathbf{r})=\frac{|e| n_{D}(\mathbf{r})}{1+\exp \left(\frac{|e| \phi_{\mathrm{tot}}(\mathbf{r})+E^{d}}{k T}\right)}
$$

which tends to distribution given by $(8)$ in $T=0$ limit. For the electron gas at the interface we use the formula

$$
\rho_{\mathrm{d}}(\mathbf{r})=\frac{\tau \rho_{\mathrm{el}}^{\mathrm{as}}(z)}{1+\exp \left(\frac{-|e| \phi_{\mathrm{tot}}\left(x, y, z_{0}\right)+U_{b}\left(z_{0}\right)}{k T}\right)},
$$

which in the limit $T \rightarrow 0$ tends to Eq. (20) with $\tau$ given in (21). We start the iteration at $T=15 \mathrm{~K}$, in which the convergence is quickly reached. Next the temperature is gradually

reduced to the nominal value. With the simulated cooling the convergence is obtained with an overall number of iterations that is reduced ten times. 


\section{RESULTS}

The numerical procedure described above was applied for the device described in Ref. [10]. Basic parameters of the nanodevice including composition and width of the semiconductor layers, the position and shape of the gates, and the applied voltages are adopted according to the experimental data. $\frac{10}{}$ The theory contains a single free parameter: the dopant concentration in the AlGaAs barrier, which is not known with a sufficient precision since the position of the transmission lines turns out to be extremely sensitive to its even small variation. The donor density appearing in formulas (8) and (28) was set to reproduce the charging of the dot with the first electron when the voltages are set as in the experiment (see below).

The two-dimensional electron gas in the asymptotic region acts as an electron reservoir whose electrochemical potential is set by the voltage of the source and drain that are connected to the electron gas in the asymptotic region. We adopt the potential applied to the source, drain and the electron gas as the reference value for the voltages $V_{S}=V_{D}=0$. Following the experiment $\frac{10}{n}$ we assume that voltages applied to the electrodes are: $V_{P}=0$, $V_{T}=1.5 \mathrm{~V}, V_{M}=-1.07 \mathrm{~V}$ and $V_{R}=-0.096 \mathrm{~V}$. Under these voltages a first electron occupies the dot. 10 Figs. $4(\mathrm{c})$ and $4(\mathrm{~b})$ present the electrostatic potential distribution in $x, y$ plane for $z=z_{o}=12 \mathrm{~nm}$ [used in Eq. (25)] calculated for $n_{D}=20 \times 10^{16} \mathrm{~cm}^{-3}$ and $n_{D}=25 \times 10^{16} \mathrm{~cm}^{-3}$, respectively. In both figures the blue line shows the zero of the electrostatic potential. Note, that in Fig. 4(c) the zero potential is found far from the center of the device which indicates that the dot cannot trap any electrons. In Fig. 4(b) the zero level encircles quite a large region which turns out to trap several electrons and not a single one. We found that a single electron occupies the dot with the zero binding energy for the donor concentration $n_{D}=21.641 \times 10^{16} \mathrm{~cm}^{-3}$. The corresponding potential profile in presented on a surface plot in Fig. 4(a) and as a contour plot in Fig. 5(a). The shaded region in Fig. 5(a) shows the electrodes. The potential has a well developed minimum of negative potential that is just enough to trap a single electron. The electron reservoir which encircles the dot is presented in Fig. 6 which shows a density map of the electron gas confined at the heterojunction. For the applied voltages the oscillating plunger gate $(P)$ voltage takes the electron out of the dot to the reservoir and attracts it back with the electron tunneling along the transmission channel that is opened parallel to the $x$ direction. The opened channel is 
(a)

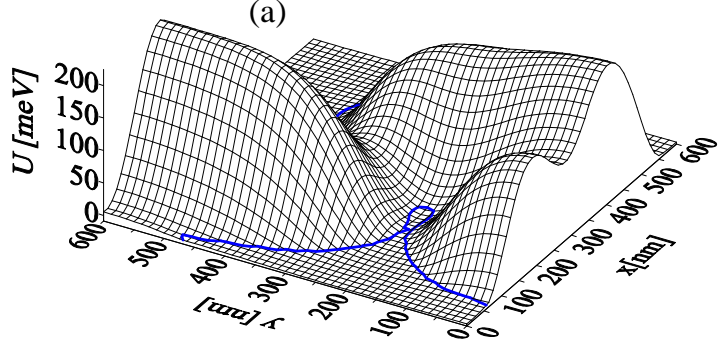

(b)

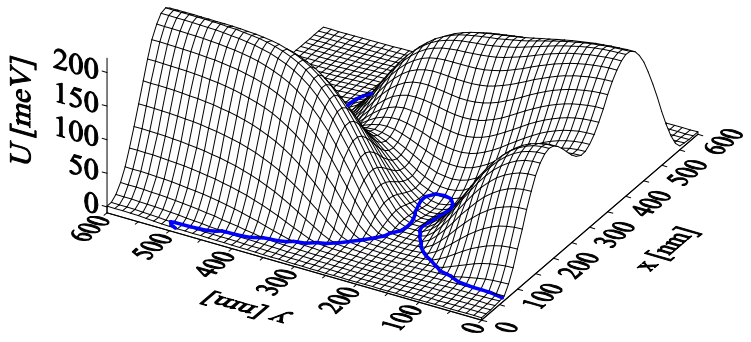

(c)

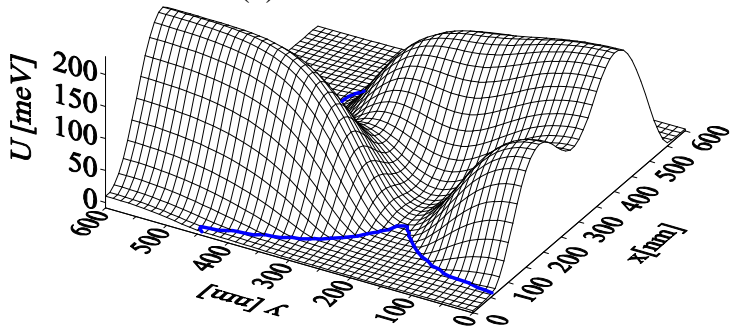

FIG. 4: Electron potential energy $U(x, y)=-|e| \phi_{\text {elst }}\left(x, y, z_{o}\right)$ calculated at a distance of $z_{o}=12$ $\mathrm{nm}$ of the AlGaAs barrier for voltages $V_{P}=0, V_{T}=1.5 \mathrm{~V}, V_{M}=-1.07 \mathrm{~V}$ and $V_{R}=-0.096 \mathrm{~V}$. Assumed donor impurity concentration is $n_{D}=21.641 \times 10^{16} \mathrm{~cm}^{-3}$ in (a), $n_{D}=25 \times 10^{16} \mathrm{~cm}^{-3}$ in (b) and $n_{D}=20 \times 10^{16} \mathrm{~cm}^{-3}$ in (c). Blue line shows the $U=0$ contour.

visible in Fig. 5(a) for $x, y \simeq 200 \mathrm{~nm}$. In Fig. 5(b) and (c) the potential cavity is surrounded by potential barriers that are too deep for the electron to cross.

Formation of a single-electron artificial atom is obtained also for other voltages. Lowered $V_{M}$ voltage results in an increase of the electron potential energy in the center of the nanodevice that can be compensated by an increase of $V_{R}$. A transmission line can be traced on the $\left(V_{M}, V_{R}\right)$ plane. Similar transmission lines are observed for the tunneling of the second electron from and into the dot. In Fig. 7 we present the transmission lines calculated for 1-4 electrons which are compared to the experimental results. 10 We note that the calcula- 

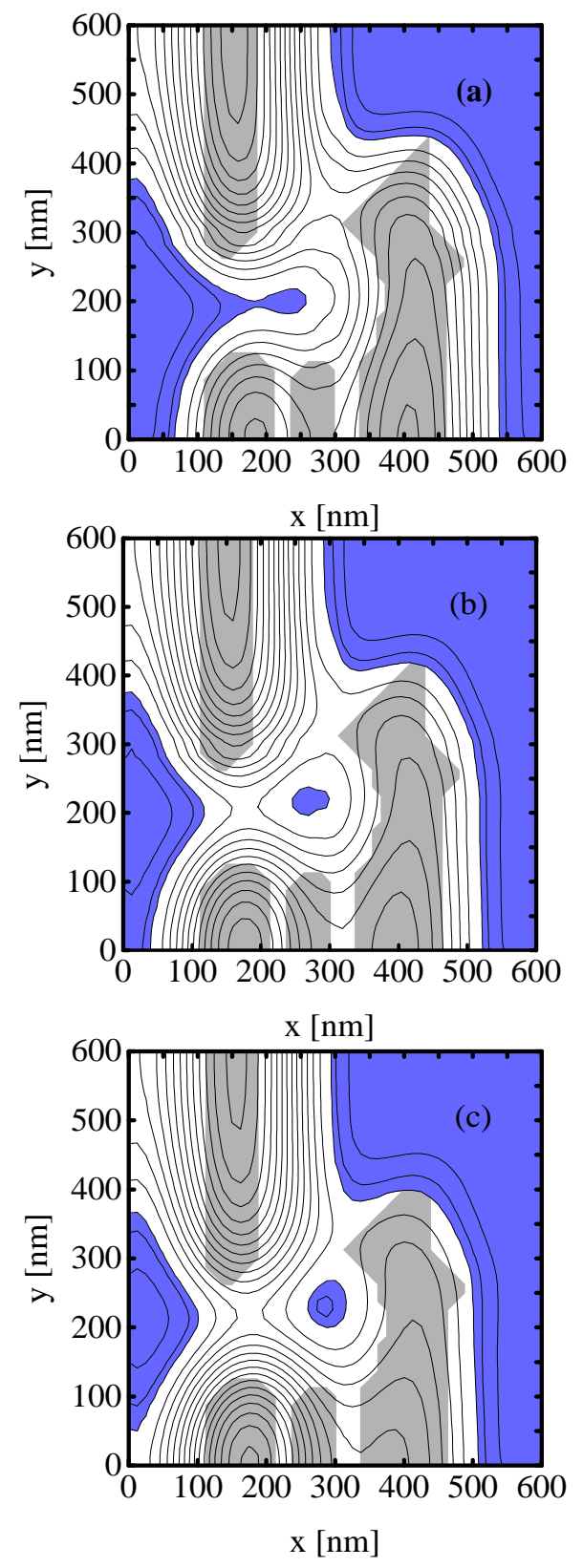

FIG. 5: (Color online) Equipotential contours and regions of negative electron potential energy (plotted in blue) for $n_{D}=21.641 \times 10^{16} \mathrm{~cm}^{-3}$ at $12 \mathrm{~nm}$ of the barrier. In (a) the voltages are same as in Fig. 4. In (b) and (c) $(-1.12 \mathrm{~V},-0.93 \mathrm{~V})$ and $(-1.18 \mathrm{~V},-0.90 \mathrm{~V})$, respectively. Shaded regions show the metal gates.

tions very well reproduce both the slope and the positions of the transmission lines. The experimental lines for the first and the second electron vanish for larger $V_{M}$, which is due to the increase of the potential barrier closing the transmission channel. For third and fourth 


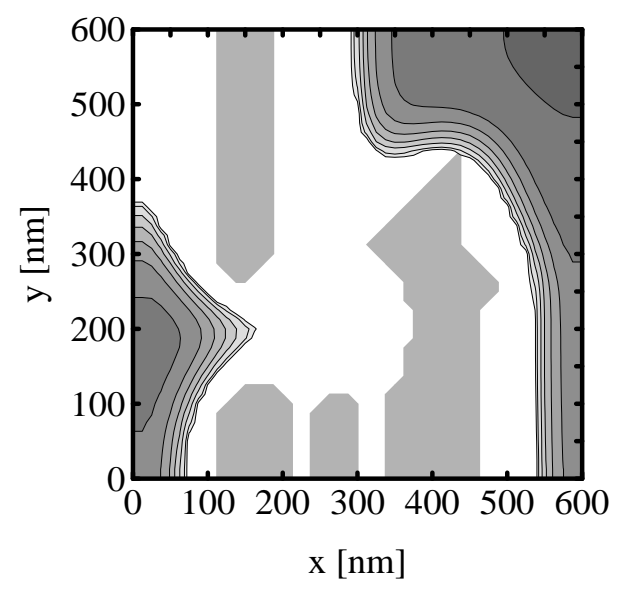

FIG. 6: Contour plot of the density of the electron gas at $12 \mathrm{~nm}$ of the barrier for the voltages used in Fig. 6(a), the darker shade of grey the larger density.

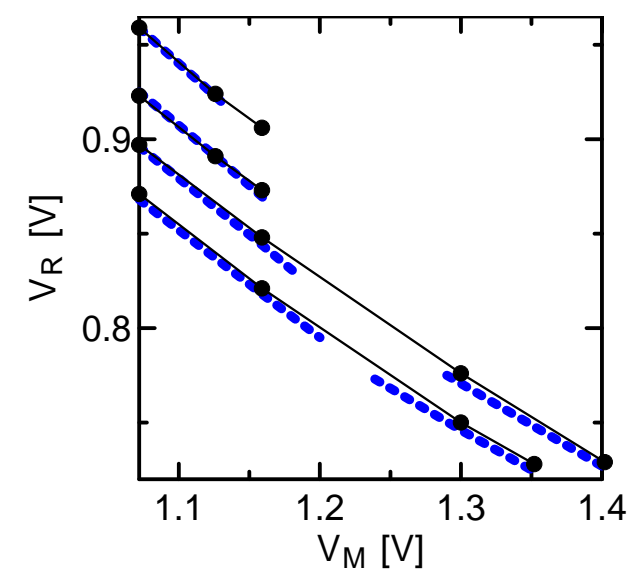

FIG. 7: Transmission lines as calculated (solid lines) and measured 10 (thick dashed lines). Above the highest line the dot is empty.

electron the lines reappear for larger $V_{M}$, which is explained by opening of a second transmission channel. Increase of the potential barrier and formation of the second transmission channel can be observed in Figs. 5(b) and 5(c) which present the potential distribution with a single dot-confined electron calculated for $V_{M}, V_{R}$ voltages equal to $(-1.12 \mathrm{~V},-0.93 \mathrm{~V})$ and $(-1.18 \mathrm{~V},-0.90 \mathrm{~V})$, respectively. Fig. 5(b) shows the case where the left channel of Fig. 5(a) is closed. In Fig. 5(c) we see lowering of the potential barrier at right of the dot center. The tunneling probability is still not large enough to allow for an experimental observation of the transmission. 
Interaction of the confined electrons with the electron gas leads to an appearance of nonlinear effects in the Schroedinger equation $\underline{30} \underline{31}$ for the dot-confined electrons. The nonlinear effects increase the electron binding energies and result in self-focusing mechanism for a single confined electron. An account of this self-focusing potential is taken in the Poisson equation (515). The Poisson equation does not account for the energy relaxation of the dot-

confined-electron system to the reservoir. $\stackrel{31}{=}$ The energy relaxation leads to a decoherence of the dot-confined quantum states. The energy relaxation rate is likely to be significant due to small density of the electron gas in the neighborhood of the dot. $\underline{31}$

\section{SUMMARY}

We presented a theory describing phenomena appearing in a multielectrode device of gated two-dimensional electron gas containing a quantum dot. In the asymptotic region of a large distance from the gated area the calculation consists in solution of a single-dimensional Poisson-Schroedinger problem for the electron gas in a strong magnetic field parallel to the semiconductor surface. Solution in the asymptotic region is used to determine the electron gas density in the three-dimensional Poisson-Schroedinger problem for the gated region with a quantum dot. The energies and charge densities of several confined electrons were calculated with a configuration interaction approach. The presented theory includes a single fitting parameter - the donor concentration. All the other device parameters: the layer structure, the shape, size and position of the electrodes as well as the applied magnetic field vector are taken of the experiment $\underline{\underline{9}} \underline{10,11}$ The slope and positions of the transmission lines were reproduced with a very good quantitative agreement with the experiment. We discussed the electric field distribution in the device for voltages corresponding to transmission lines observed in Ref. [9] with a particular attention to the inhomogeneities creating the quantum dot confinement and the surrounding potential barriers that separate the artificial atom of the electron gas.

For voltages corresponding to the transmission lines the weakest bound electron is stimulated to oscillate between the dot and the reservoir by the oscillating plunger voltage. We demonstrated opening and closing of two transmission channels in the barriers that allow for the oscillations of the confined charge. 


\section{Acknowledgments}

This work was supported by the State Committee for Scientific Research (KBN) under Grant No. 1P03B 00227.

1 D.P. DiVincenzo, Phys. Rev. A 51, 1015 (1995).

2 D. Loss and D.P. DiVincenzo, Phys. Rev. A 57, 120 (1998).

3 R.C. Ashoori, H.L. Stormer, J.S. Weiner, L.N. Pfeiffer, S.J. Pearton, K.W. Baldwin, and K.W. West, Phys. Rev. Lett. 68, 3088 (1992).

4 S. Tarucha, D.G. Austing, T. Honda, R.J. van der Hage, and L.P. Kouwenhoven, Phys. Rev. Lett. 77, 3613 (1996).

5 R.C. Ashoori, N.B. Zhitenev, L.N. Pfeiffer, and K.W. West, Physica E 3, 15 (1998).

6 L.P. Kouwenhoven, T.H. Osterkamp, M.W.S. Danoesastro, M. Eto, D.G. Austing, T. Honda, and S. Tarucha, Science 278, 1788 (1997).

7 S. De Franceschi, S. Sasaki, J.M. Elzerman, W.G. van der Wiel, S. Tarucha, and L.P. Kouwenhoven, Phys. Rev. Lett. 86, 878 (2001).

8 M. Rontani, C. Cavazzoni, D. Bellucci, and G. Goldoni, J. Chem. Phys. 124, 124102 (2006).

9 R. Hanson, B. Witkamp, L.M.K. Vandersypen, L.H. Willems van Beveren, J.M. Elzerman, and L.P. Kouwenhoven, Phys. Rev. Lett. 91, 196802 (2003).

10 J.M. Elzerman, R. Hanson, L.H. Willems van Beveren, L.M.K. Vandersypen, and L.P. Kouwenhoven, Appl. Phys. Lett. 84, 4617 (2004).

11 J.M. Elzerman, R. Hanson, L.H.Willems van Beveren, B. Witkamp, L.M.K. Vandersypen, and L.P. Kouwenhoven, Nature 430, 431 (2004).

12 R. Hanson, L.H. Willems van Beveren, I.T. Vink, J.M. Elzerman, W.J.M. Naber, F.H.L. Koppens, L.P. Kouwenhoven, and L.M.K. Vandersypen, Phys. Rev. Lett. 94, 196802 (2005).

13 T. Meunier, I.T. Vink, L.H. Willems van Beveren, F.H.L. Koppens, H.P. Tranitz, W. Wegscheider, L.P. Kouwenhoven, and L.M.K. Vandersypen, Phys. Rev. B 74, 195303 (2006).

14 J.R. Petta, A.C. Johnson, J.M. Tylor, E.A. Laird, A. Yacoby, M.D. Lukin, C.M. Marcus, M.P. Hanson, and A.C. Grossard, Science 309, 2180 (2005).

15 J.M. Elzerman, R. Hanson, J.S. Greidanus, L.H.Willems van Beveren, S. De Franceschi, L.M.K. 
Vandersypen, S. Tarucha and L.P. Kouwenhoven, Physica E 25, 135 (2004).

16 J. Kim, D.V. Melnikov, J.P. Leburton, D.G. Austing, and S. Tarucha, Phys. Rev. B 74, 035307 (2006).

17 W.G. van der Wiel, S. De Franceschi, T. Fujisawa, J.M. Elzerman, S. Tarucha, and L.P. Kouwenhoven, Science 289, 2105 (2000).

18 R. Hanson, L.H. Willems van Beveren, I.T. Vink, J.M. Elzerman, W.J.M. Naber, F.H.L. Koppens, L.P. Kouwenhoven, and L.M.K. Vandersypen, Phys. Rev. Lett. 94, 196802 (2005).

19 R. Hanson, B. Witkamp, L.M.K. Vandersypen, L.H. Willems van Beveren, J.M. Elzerman, and L.P. Kouwenhoven, Phys. Rev. Lett. 91, 196802 (2003).

20 N.A. Bruce and P.A. Maksym, Phys. Rev. B 61, 4718 (2000).

21 S. Bednarek, B. Szafran, and J. Adamowski, Phys. Rev. B 61, 4461 (2000).

22 S. Bednarek, B. Szafran, and J. Adamowski, Phys. Rev. B 64, 195303 (2001).

23 B. Szafran, S. Bednarek, and J. Adamowski, Phys. Rev. B 67, 115323 (2003).

24 P. Matagne, J.P. Leburton, D.G. Austing, and S. Tarucha, Phys. Rev. B 65, 085325 (2002).

25 P. Matagne and J.P. Leburton, Phys. Rev. B 65, 235323 (2002).

26 S. Bednarek, B. Szafran, K. Lis, and J. Adamowski, Phys. Rev. B 68, 155333 (2003).

27 D.V. Melnikov, P. Matagne, J.P. Leburton, D.G. Austing, G. Yu, S. Tarucha, J. Fettig, and N. Sobh, Phys. Rev. B 72, 085331 (2005).

28 L.X. Zhang, P. Matagne, J.P. Leburton, R. Hanson, and L.P. Kouwenhoven, Phys. Rev. B 69, 245301 (2004).

29 A. Weichselbaum and S.E. Ulloa, Phys. Rev. B 74, 085318 (2006).

30 S. Bednarek, B. Szafran, and K. Lis, Phys. Rev. B 72, 075319 (2005).

31 S. Bednarek and B. Szafran, Phys. Rev. B 73, 155318 (2006).

32 In Ref. 27 calculations for a rectangular vertical dot were performed.

33 In the limit of small mesh spacings $(\Delta x, \Delta y, \Delta z)$ the value of $z_{0}$ has no influence on the energy of confined system, which can be verified for the single-electron case. In the few-electron calculations the spacings cannot be made very small. For a single electron the results obtained with $z_{0}$ adopted variationally are consistent with the zero mesh spacing limit. 